\title{
Phase diagram of dilute polyelectrolytes: Collapse and redissolution by association of counterions and coions
}

\author{
Francisco J. Solis \\ Department of Physics and Astronomy, Arizona State University, P.O. Box 87-1504, Tempe, AZ 85287.
}

Francisco.Solis@asu.edu

\begin{abstract}
Dilute solutions of strongly charged polymer electrolytes undergo, upon addition of multivaltent salt to the solutions, a phase transition from extended conformations to collapsed or bundled ones. Upon further addition of salt they experience a second transition, a redissolution back into extended conformations. This paper presents a theoretical study of the structure and properties of the phase diagram of these solutions. On the basis of simple phenomenological observations a schematic phase diagram is constructed that allows a simple and explicit determination of the direction of the tie lines within the coexistence region. The actual shape of the coexistence boundary is determined by means of a model mean free energy functional that explicitly includes the possibility of association of both counterions and coions to the electrolyte. It is found that it is possible to redissolve the electrolytes into conformations where the bare charge of the electrolyte is overcompensated by the counterions but, due to the associated coions, can have either sign of total effective charge. When coion association is possible, the redissolution approximately coincides with the reassociation of the coions and counterions in the bulk of the solution.
\end{abstract}

\section{INTRODUCTION}

Dilute solutions of strongly charged polymer electrolytes (polyelectrolytes) such as single and double stranded DNAl 1 , polystyrene sulphonate ological and synthetic polymeric systems卧 exhibit phase and/or structural transitions upon addition of multivalent salts to the solutions. First, the addition of multivalent salt causes a collapse of the extended structure of flexible polyelectrolytes or the bundling in mono- or multi- molecular structures of semiflexible and rigid electrolytes that segregate into a dense phase, a phenomenon which in the case of DNA is also know as DNA condensation. Upon further addition of salt, the electrolytes redissolve acquiring again extended or unbundled states. There are many different examples of this type of system and it is clear that while the strong charge of the electrolytes is required for the transitions, the precise geometry of the polymers is not a crucial factor. The fact that the transitions are driven only by a combination of electrostatic interactions with entropic and sterif effects has been shown by many computer simulations. 40

The first transition to a collapsed state is explained by the onset of attractive interactions between almost neutral structures formed by electrolytes whose charge is almost compensated by associated multivalent counterions. 113 The redissolution transition indicates that as the concentration of multivalent salt increases the system finds more energetically favorable conformations without packaging its structure. The model presented in this paper emerges from two previously proposed ideas regarding the redissolution transition. In a previous work 14 it was argued that a determining factor for the redissolution is the nature of the salty environment into which the electrolyte redissolves. In particular, it was emphasized that at large multivalent salt concentrations the thermodynamics of the multivalent counte- rions and monovalent coions is affected by the Bjerrum association 15 where the ions of the salt within the solution become strongly correlated with each other. Thus, an scenario for the redissolution is that the multivalent ions associated with the electrolyte prefer, without leaving the polymer, to be exposed to such environment. On the other hand, it has been argued by the group of Shklovskiil 13 that the main reason for the redissolution is the decrease in energy available to the system by overcharging the electrolytes 16 . Since the collapsed structure has to be effectively neutral, the electrolytes redissolve so that more counter-ions can associate to them. Both these ideas can be combined and the result provides an interesting picture of different possible charged states for the redissolved electrolyte.

The central point of this paper to exhibit, by means of a simple model, that for large amounts of multivalent and monovalent salt added to the system, the redissolution transition is marked by the onset of reassociation of the multivalent salt ions on the bulk of the solvent. In its redissolved state the electrolyte acquires a large number of multivalent counter-ions that overcompensate its bare charge but, also, associated with them, a large number of coions that form a second associated layer. Due to the large amount of screening of the electrostatic interaction near redissolution conditions, the decrease in energy due to the reassociation of coions with the multivalent counterions at the electrolyte surface is of magnitude similar (but smaller) to the energy of reassociation in the bulk of the solution. Thus, this second layer of associated coions can only exist if Bjerrum association is already present in the bulk of the solution. This second layer enhances the decrease in energy of the electrolyte due to the overcharging of the first layer. The presence of the coions reduces the net charge of the region near the electrolyte and thus decreases the Coulombic barrier to multivalent ions overcharge. Furthermore, in such a state, 
there is a greater number of particles in strongly correlated states thus decreasing the energy per electrolyte and making its redissolution more favorable. The total charge of the near region of the electrolyte that contains all these associated charges can have either charge sign, but in all cases the charge from the multivalent ions overcompensates the bare charge of the electrolyte. This multiple-species association has been observed in recent computer simulations by Tanaka and Grosberget where the electrolyte is a small colloidal sphere and by Holm and Kremerls where the electrolyte is a rigid rod 18 . The possibility of this second associated layer has been known for long time 19,20 but its implications for these phase transitions has not been fully explored. Figure 1 presents an sketch of different possible charged states of the near region of an electrolyte. Consider, for example, a section of electrolyte with total bare charge -12 , and multivalent counter-ions of charge $z=+3$. At low amounts of multivalent-salt the total charge of the system is negative, but partially compensated by (a) positive monovalent or (b) multivalent ions. The collapsed state (c) is neutral with the electrolyte charge compensated by positive multivalent ions, where different electrolytes or sections of electrolytes share the positive charges. In the redissolved state the charge of the electrolyte is overcompensated by multivalent ions but the total associated charge can be (d) positive, (e) neutral, or (f) negative due to the association of a second layer of negative ions.

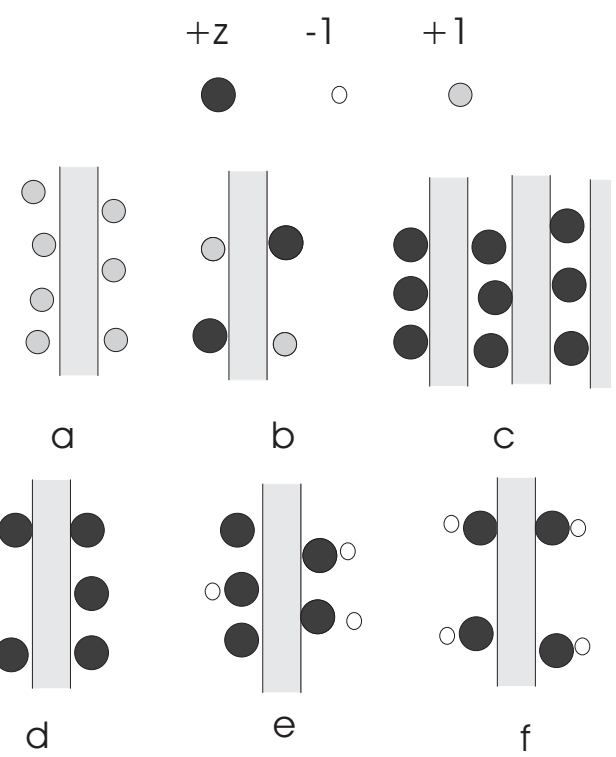

FIG. 1. Sketch of different charge states of a rod-like electrolyte with associated counterions and coions. At low amounts of multivalent-salt the total charge of the system is negative, but partially compensated by (a) positive monovalent or (b) multivalent ions. The collapsed state(c) is neutral with the electrolyte charge compensated by positive multivalent ions. In the redissolved state, the total charge can be (d) positive, (e) neutral, or (f) negative.

A second goal of this article is to present a simple con- struction of the phase diagram of the multi-species electrolyte system. While conditions for collapse or redissolution implicitly assume some form of phase transition its global structure has not been previously considered in detail. This article proposes, on the basis of simple phenomenological observations, a construction of the structure of the phase diagram, the location of the coexisting phases, and the slopes of tie-lines.

In the rest of the paper, all free energies are measured in units of $k T$, with $k$ the Boltzmann constant and $T$ the temperature. All concentrations are presented as number densities. Lengths are measured with respect to a microscopic length unit $a$ that is the average size of monomers of the polymer and ions, which are assumed to be of roughly the same size. The strength of the electrostatic interaction is quantified by the Bjerrum number $B=e^{2} / \varepsilon a k T$ which is energy of interaction of two elementary charges at its nearest possible distance in water (or the medium considered).

\section{STRUCTURE OF THE PHASE DIAGRAM.}

\section{A. Boundaries of the phase diagram}

The basic components of the system are: a solvent, a dilute polyelectrolyte with monomer concentration $\phi$, monovalent salt $s$ and a multivalent salt $\rho$. Since each of these components dissociate into different ions, we actually start with six different ionic components in solution. To reduce this number, it is possible to consider the case in which the charges of the ions are -1 for each monomer of the polymer, +1 for the original counterions of the polymer, -1 and +1 for the ions of the monovalent salt, and $+z$ and -1 for the ions from the multivalent salt. It is further assumed that all of the +1 ions are identical, and similarly for the -1 ions. Thus there are only four components, besides the solvent: dissociated polymer at concentration $p=\phi$, positive multivalent ions $m=\rho$, positive monovalent ions $t=\phi+s$, and negative monovalent ions $u=z m+s$. A further constraint is that each phase formed has to be electroneutral so that the condition $-p+z m+t-u=0$ is always satisfied. These restrictions allow for a description of all the ionic species in terms of only three concentrations, either of salts $(\phi, \rho, s)$, or ions $(p, m, t)$.

The allowed values of the concentrations of ions can be described as follows. Each ionic particle density should be non-negative, and in the reduced representation $(p, m, t)$ the requirement $u \geq 0$ becomes the constraint $t-p+z m \geq 0$. Carrying this constraint to the salt representation shows that $(\phi, \rho, s)$ should satisfy

$$
\begin{aligned}
0 & \leq \phi, \\
0 & \leq \rho, \\
-\min (z \rho, \phi) & \leq s .
\end{aligned}
$$


This last inequality has the following interpretation. Consider the case of a state with a small amount of solvent and a dense mixture of only polymer ions with multivalent ions, so that $(p, m, t)=(x,-x / z, 0)$ (and $u=0)$. This state is physically realizable but its description in terms of the salt variables is $(\phi, \rho, s)=(x, x / z,-x)$. To obtain this state one can start with a mixture of polyelectrolyte and multivalent salt and then extract monovalent salt, that is, extract both positive monovalent counterions (given by the polyelectrolyte), and negative monovalent ions (given by the salt). The boundaries of the possible states of the system in the salt variables representation are sketched in Figure 2.

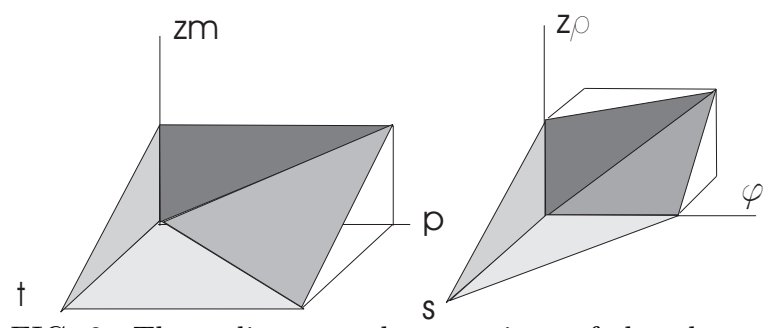

FIG. 2. These diagrams show sections of the planes that bound the region of allowed states for the system in the $(p, m, t)$, and $(\phi, \rho, s)$ representations. As explained in the text, the allowed states extend into negative values of the monovalent salt variable $s$.

\section{B. Location of the dense phase}

Experiments have shown the phase segregation of neutral complexes with little amount of solvent from the bulk of the dilute polyelectrolyte solution. The precise location of this dense phase in the salt or ionic components diagram has not been precisely determined experimentally. Here it is only necessary for our purposes to assume that this phase has zero or very small amount of monovalent ions present and that is rich in polyelectrolyte neutralized with multivalent ions with a very small amount of solvent. The dense phase is not a unique point in the phase diagram, but it can be described as a region in the neighborhood of the point $(p, m, t)=(\Phi, \Phi / z, 0)$, or $(\phi, \rho, s)=$ $(\Phi, \Phi / z,-\Phi)$, where $\Phi$, is the (large) concentration of monomers in this phase and, as explained above, the negative value of $s$ reflects the depletion of monovalent ions from these states. To simplify the model, however, it can be assumed that the dense phase consists of only one point, namely $(\phi, \rho, s)=(\Phi, \Phi / z,-\Phi)$.

\section{The tie lines}

The precipitation of the collapsed neutral structures from the dilute solution indicates that the system has entered into a coexisting phase. A point $(\phi, \rho, s)$ inside the coexistence region decomposes into two coexisting points with compositions $(\Phi, \Phi / z,-\Phi)$, namely the dense state, and a second polymer dilute point of the form $(\phi-x \Phi, \rho-x \Phi / z, s+x \Phi)$. The assumption that most of the high polymer density points of the region of coexistence lie in a very small region and are modeled as one single point immediately gives rise to this specific decomposition, and it is clear that the slope of the tie-lines is always determined by this construction.

To summarize, the phenomenological observation that the dense polymer phase is neutral with the polyelectrolyte charge neutralized by multivalent salts leads to a phase diagram of the form shown in Figure 3 including the tie lines shown. (The choice of cross section for different values of the $t$ variable are meant to aid visualization of the diagram and do not reflect actual energetic considerations. An example of actual cross-sections in logarithmic variables is presented in the Figure 4).

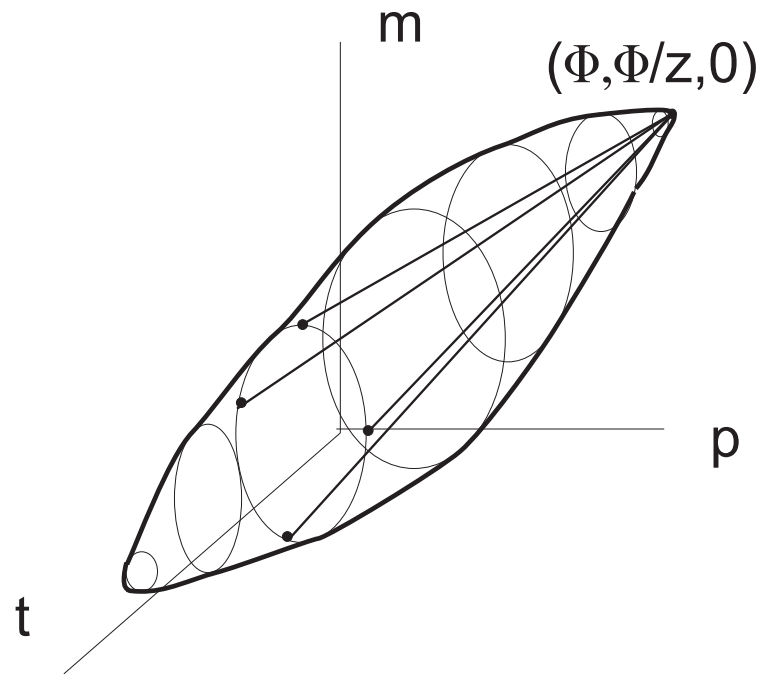

FIG. 3. Scheme of the region of coexistence in the reduced description of the components of the system. It is assumed that all tie lines connect points of the bounding surface of the coexistence region with a single point $(\Phi, \Phi / z, 0)$ at the one extreme of the coexistence region. Four tie lines are shown for the points corresponding to a section defined by a constant value of the $t$ component.

\section{FREE ENERGY FUNCTIONAL}

To be able to write down an effective free energy functional that describes the polyelectrolyte phase transition it is necessary to consider coherently but separately the contributions to the free energy from three different regions. There is a dense precipitated phase that is relatively poor in solvent and rich in polymer and multivalent ions. Within the dilute phase, there are two distinct regions: the one occupied by the polymer and its associated charges, and the region away from the polymer occupied only by solvent and ions.

There are many variants of the problem all of which present similar behavior. The same type of phase diagram occurs for flexible and rigid polyelectrolytes of dif- 
ferent lengths. It is simplest to write a model for a collection of rigid rods (say short or medium size DNA). In this case the main barrier arising from the structure of the polyelectrolyte to the formation of a collapsed state is purely entropic. If one considers very long semiflexible molecules, the collapse is monomolecular and the barrier is of a elastic nature instead of entropic, and requires the introduction of further parameters. The results are nevertheless similar.

\section{A. Two States modeling}

While it is clear that the region of phase coexistence does not separate two topologically disconnected regions in the full phase diagram, it is nevertheless useful to write down two separate free energy functionals for the states at the ends of a tie line. It is very hard to write down one single free energy functional that interpolates between the dense and dilute phase, especially for flexible electrolytes. When using different functionals for each phase, a phase transition can be properly determined only if both functionals are good approximations of the absolute free energy and refer to a same (arbitrary) zero energy point. In this case this goal is achieved by using similar approximations in the calculation of the free energy in the two states.

\section{B. The Dense Phase}

In the dense phase, the charges are very close to each other although it is likely that they retain a large amount of water. This phase is electroneutral and effectively forms a bulk region where the all the charges are strongly correlated with their near neighbors. In previous work 12 it was argued that a suitable free energy per unit volume $F_{d}$ for this state, is given by:

$$
F_{d}(\Phi, \Phi / z,-\Phi)=-M \Phi
$$

where $M$ in an effective Madelung constant that depends on the specific geometry of the polymer and ions used but that reflects the strongly correlated state of the charges in that phase.

\section{The dilute region}

The dilute phase is not homogeneous at a microscopic level. The charge distribution is very different near the polyelectrolyte compared to regions away from it. The free energy of this phase can be approximated by separating a region near the polymer from the rest of the solvent. Since the polymer concentration is very dilute, this region can be considered as a separate thermodynamic system in equilibrium with an ion reservoir. The amount of charge per monomer associated to the polymer from each of the ionic species is $f_{m}, f_{u}, f_{t}$. The average ionic concentrations in the bulk of the solution excluding regions occupied by polymer is then $(m, t, u)_{e f f}=\left(m-f_{m} p / z, t-f_{t} p, u-f_{u} p\right)$.

The free energy density for the system polyelectrolyte plus solvent can be written as:

$$
F_{t}=\frac{\phi}{N} \ln \frac{\phi}{N}+\phi F_{e}+F_{s}
$$

where $F_{t}$ is the total free energy density in the dilute phase, $F_{e}$ is the energy per electrolyte monomer due to the interactions between its own and associated charges, and $F_{s}$ is the energy of the solvent an the ions in solution. The values of the associated fractions $f_{i}$ are determined by minimization of this expression with respect to them.

The salty environment can be modeled as an ionic solution obeying a Debye-Huckel free energy where it is necessary to consider not only the multivalent positive, monovalent positive and negative ions but also an extra species, the reassociated ions. At the typical concentration at which the redissolution of the electrolyte takes place, there are important corrections to the free energy of the solution due to this phenomenon. The simplest way to describe the association is to assume that a finite fraction $f_{b}$ of all the multivalent ions in solution are associated with the same number of positive monovalent ions thus forming a new species of reduced charge $(z-1)$ with concentration $m_{a}=f_{b} m_{e f f}$. While in general there can be further reassociation to states of charge $z-2$ etc., only the first reassociation will be treated explicitly here. Upon reassociation, the magnitude of the energy of a pair of associated ions is $g_{b}$. The new concentrations of non associated ions are $m_{n}=m_{e f f}-m_{a}$, and $u_{n}=u_{e f f}-m_{a}$. The free energy for the solvent is then:

$$
F_{s}=\sum \psi_{i} \ln \psi_{i}-\frac{1}{12 \pi} \kappa^{3}-g_{b} m_{a}
$$

where $\kappa$ is the screening length of the system and is given by

$$
\kappa^{2}=\sum_{i} 4 \pi z_{i}^{2} \psi_{i}
$$

where the ionic densities $\psi_{i}$ with charges $z_{i}$ are $m_{a}, m_{n}$, $t_{e f f}$ and $u_{n}$.

The free energy of the near region of the polyelectrolyte, per monomer of electrolyte can be expressed as an expansion up to quadratic order on the fractions of associated charges. A good choice for this functional is

$$
F_{e}=\frac{G_{2}}{2} q^{2}-g_{m} f_{m}-g_{t} f_{t}-g_{u} f_{u}+g_{c} f_{m} f_{u} .
$$

To argue for such functional it is convenient to separate within the near region of the electrolyte, the contributions to the free energy coming from a small region near each positive multivalent or monovalent ion, and the interaction of between these ions with others further away. 
The long range screened interaction between charges gives rise to the quadratic contribution $G_{2} q^{2} / 2$, where the effective charge per monomer is $q=1-f_{m}+f_{u}-f_{t}$. The coefficient of interaction depends on the geometry of the system and for simplicity is chosen to have the form $G_{2}=G_{1} B \ln ((1+\kappa) / \kappa)$, where $G_{1}$ is a geometric factor. For large screening lengths $\kappa^{-1} \gg 1$, this term reflects the interaction of each charge with the logarithmic field of a charged rod of length $\kappa^{-1}$. At very small screening lengths $\kappa^{-1}<1$ the function goes to 0 .

When the effective electrolyte charge is near zero the linear terms in the free energy reflect the correlation energy of each charge with its near neighbors. At zero effective charge, the amount of energy gained from the addition of a unit charge from a given ion is simply $-g_{m},-g_{s}$, or $-g_{t}$. The association of a positive monovalent charge has as prerequisite the presence of multivalent negative ions associated to the electrolyte, so that $f_{u}<f_{m}$. The reduction in energy for this association is required to be comparable (but smaller) to the energy of association for these pairs of ions in the bulk so that $g_{u}<g_{b}$ the positive charge is brought into contact with a positive associated ion but it is also in the neighborhood of the negatively charged polyelectrolyte.

The reassociation of the multivalent ions and negative coions influences the strong correlations between associated multivalent ions. As a larger number of monovalent ions enter the near region, the multivalent ions reduce its correlations since their repulsion is diminished by the presence of the negative ions associated with them. This element is reflected in the correlation reduction term $g_{c}$. More such terms describing corrections to the correlations can be written but it turns out that the inclusion of just this one is sufficient to recover a phase diagram that is similar to those observed in experiments. Omission of this term leads to much more extended regions of coexistence.

\section{DETERMINATION OF COEXISTENCE REGIONS}

\section{A. Equilibrium between phases}

A state with concentrations $(p, m, t)$ decomposes into two states if the following free energy, for some value of $x, 0 \leq x \leq 1$, is smaller than the total energy of that state.

$F_{\text {comp }}=x F_{d}+F_{t}(\phi-x \Phi, \rho-x \Phi / z, s+\Phi) \leq F_{t}(\phi, \rho, s)$

The boundary of the coexistence region is determined by points at which the condition above is an equality and the required value of $x$ is zero. Expansion of $F_{\text {comp }}$ with respect to $x$ leads to

$$
-M-\mu<0
$$

where the effective chemical potential pre monomer for the addition of a neutral electrolyte complex into the dilute region is

$$
\mu=\partial_{\phi} F_{t}+(1 / z) \partial_{\rho} F_{t}-\partial_{s} F_{t}
$$

On the basis of the assumption made, the final condition for the determination of the coexistence boundary does not explicitly depend on the concentrations in the dense phase given by $\Phi$, but only on the bulk energy per electrolyte charge $-M$ and the slope of the tie-line. The specific value of $\Phi$ is, nevertheless, important to determine the condensed electrolyte fraction $x$.

\section{B. Collapse}

In a typical experiment performed to study the phase transitions of this type of system, the amount of multivalent salt present is changed from 0 to some maximum value in small increments. The first transition occurs (for low monovalent salt) when the amount of charge provided by the multivalent salt is approximately the same as the amount of polyelectrolyte. If there is a large amount of monovalent salt, the transition requires a larger amount of multivalent salt. Thus, it is possible to approximate the location of the transition as follows: the condition Eq.9 is satisfied almost as soon as full charge compensation of the electrolyte by multivalent ions occur. The chemical potential $\mu_{m}$ of the ions in the solution, disregarding the Debye Huckel contribution, is $\ln \left(m-f_{m} \phi\right)$ with $f_{m} \approx 1$. Thus, for low monovalent salt concentrations the condition is simply that there must be enough ions to compensate the bare charge of the electrolyte:

$$
z m \approx \phi
$$

At larger monovalent salt concentrations, the multivalent ions must first displace associated positive monovalent ions from the electrolyte. The reduction in the energy from the association of the monovalent and multivalent ions is, per charge, approximately the same and thus the chemical potential in the solvent for the multivalent ions should be smaller than that of the monovalent ions. The condition for the charging of the electrolyte with multivalent ions, and a first approximation to the collapse transition condition is:

$$
\frac{1}{z} \ln m \approx \ln (s+\phi) .
$$




\section{Redissolution}

In solutions where even at large salt concentrations the free energy of the ions is dominated by their translational entropy and there is little correlation between charges, the redissolution transition occurs when the decrease in energy per electrolyte due to multivalent ions overcharge is larger than the bulk correlation energy of the dense phase. However, if many monovalent ions are present, the onset of association of the positive monovalent with negative multivalent ions marks a sharp decrease in the energy of the electrolyte. Not only there is a decrease in the energy of the positive charges but also it is possible to associate many more ions. The repulsive interaction is decreased as the near region can be near neutral while having acquired large number of positive ions.

To first approximation, the onset of the association of positive ions occurs when the magnitude of the translational entropy of the free monovalent ions is comparable to the energy of association $g_{b}$. At larger monovalent ion concentrations, the charging of the electrolyte by positive multivalent ions together with monovalent coions occurs when the energy of association $g_{b}$ is capable of compensating the translational entropy of the monovalent ions. Thus we have

$$
\ln u=\ln (z m+s) \approx-g_{b}
$$

This expression provides a rough approximation for the location of the second transition. For fixed amounts of added monovalent salt, the transition location is approximately a horizontal line in the $(p, m)$ or $(\phi, \rho)$ plane.

\section{A numerical example.}

The results of a numerical minimization of the model free energy appear in Figure 3. There it is shown, for different values of the monovalent salt concentration, the predicted regions of coexistence in the $(\phi, \rho)$ plane. The parameters used for this example are $B=3.0, g_{m}=9.0$, $g_{u}=9.0, g_{t}=9.6, g_{b}=1.5, g_{c}=1.8, M=9.05$. The curve containing points $a$, and $b$ has the lowest amount of added monovalent salt $s=1.810^{-5}$ (intended to represent a $0.01 \mathrm{M}$ molar concentration), the other two curves are for concentrations $s=9.010^{-5}$, and $s=1.810^{-4}$ (0.05 M, and 0.1 M). The largest salt concentration leads to smaller coexistence regions. The marked pairs of points $(a, b),(c, d)$, and $(e, f)$ correspond to the location of the pairs of collapse-redissolution transitions that occur when for fixed amounts of monovalent salts and fixed amounts of polymer, multivalent salt is added to the system.

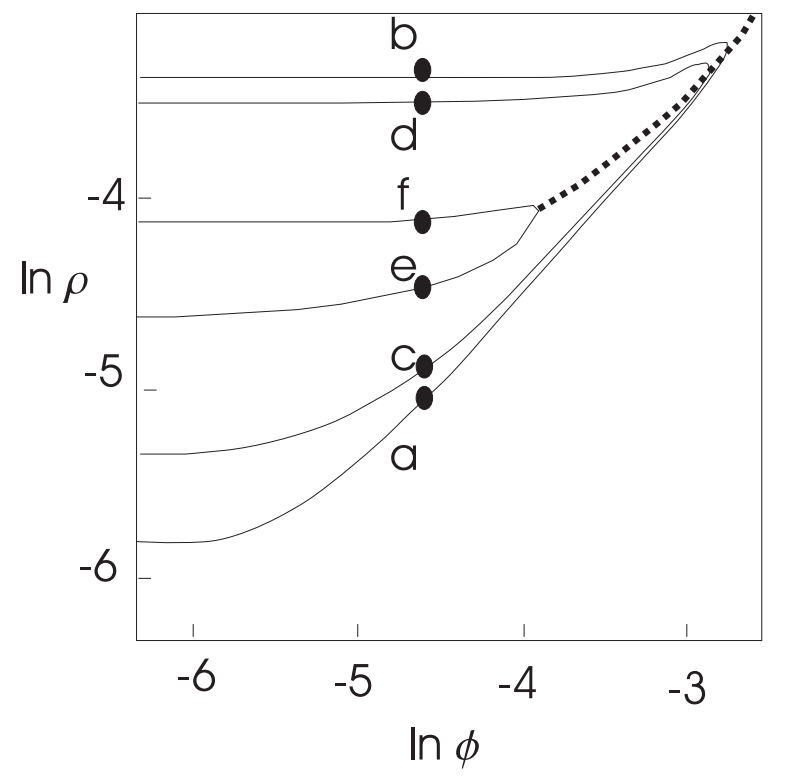

FIG. 4. The three solid curves correspond to the coexistence region boundary for three sections of the phase diagram in the $(\phi, \rho, s)$ presentation, for different amounts of monovalent salt, details in text. The largest amount of salt produces the smallest coexistence region. All states outside the boundary for each section have a negative net charge. For the case with largest amount of monovalent salt the dotted curve marks the border of the region where the multivalent ions overcompensate the bare charge of the electrolyte.

In this example, at the boundary of the redissolved region for the different values of monovalent salt added, the states $d, e$ and $f$ are all overcharged with positive multivalent ions but the net charge of all these states is negative. For the choice of parameters made, all states of the system that are not decomposed (lying outside the coexistence curve for fixed amount of monovalent salt) have a total negative charge. Different choices of parameters can lead to different charged states and, for example, in systems where the energy of reassociation of the multivalent salt is very small, all the dissociated states should have a positive effective charge, and the phase diagran has the shape proposed in work of Nguyen an Shklovskii21, where the lines of redissolution and collapse do not join to form a bounded coexistence region. The model presented here also predicts that there is a maximum value of monovalent salt for which there is a coexistence region. As in the scheme of Figure 2, the coexistence region has a limited extent in the $s, t$ or $u$ axes.

For the case of largest amount of monovalent salt added, (with coexistence curve containing the points $(e, f))$, the dotted line divides the region of states that, while effectively negative, have enough multivalent ions to overcompensate the electrolyte bare charge. Separating curves for other salt concentration show similar behavior, always originating near the "tip" of the diagram. The coexistence region sections are closed regions but their "left" boundaries lie at very small concentrations that are typically experimentally inaccessible, and are 
not shown in Figure 4.

In extremely dilute solutions there is no large difference between the original concentration of ions in the bulk of the solution and the effective concentrations obtained by subtracting the associated ions. As the amount of polymer increases, the depletion from the bulk reduces the amount of available ions and states with similar amounts of associated ions appear only at higher multivalent salt concentrations. This leads to an upward curving trend in the redissolution curves.

\section{CONCLUSIONS AND FUTURE WORK}

It is still an important but unfinished problem to refine the description of the layer of associated ions by both providing a concrete definition of the layer and by testing possible (mean field) models of the free energy contribution of the layer to the total energy of the system. In the context of integral equation approaches this goal can be achieved by locating specific properties of the ion distribution functions 18 , but ideally such definition ought to be model independent and experimentally useful. Nevertheless, even with arbitrary definitions of the layer, a free energy expansion of the form Eq. 8 is a useful instrument for the analysis of this type of system.

One would like to reduce the large number of parameters used in the present description of the system as many properties of real systems cannot be precisely measured or have a poor experimental definition. To use the ideas presented here in the context of the real system requires the collapse of information about the interaction between molecules and monomers into a few simple constants. These quantities reflect the average radii of ions and are supposed to include the steric effects of water as well as the specific location of charges in the electrolytes. A careful parametrization of simulation results, on the basis of the charge of the near region of an electrolyte was for example, presented in the work of Messina et al.22. In model systems studied though simulations or integral equations is possible to reduce the number of these parameters and calculate them on the basis of simpler geometric parameters. For comparison with experimental data it is, however, preferable to retain these constants as adjustable parameters.

A couple experimental tasks are suggested by this work as important to improve our understanding of these systems: the presentation of the data available from the point of view of phase separation of ionic species (instead of salts), a more systematic mapping of the effective charge of the electrolytes in the redissolved region of the phase diagram, and determination of the ionic composition of the associated layer (in this respect there has been progress by means of osmotic pressure measurements23 26). Future analysis of experimental data using the model presented in this article should provide a map, even if only a rough one, of the properties of specific ionic species with the parameters used. Finally, the addition of further ingredients that can directly modify the chemical potential of the different charged species should also induce dramatic changes in the location a of the transitions. From the point of view of the approach presented here, these modifications would be reflected in changes of the phenomenological parameters used in the model.

\section{ACKNOWLEDGEMENTS}

The author thanks many useful talks with Monica Olvera de la Cruz, Eric Raspaud, Boris Shklovskii, Toan Nguyen, Motohito Tanaka, Mark Stevens, and John Widom.

${ }^{1}$ The original work on collapse of DNA appears in: J. Widom, and R. L. Baldwin, J. Mol. Biol. 144, 431 (1980), Biopolymers 22, 1595 (1983).

${ }^{2}$ D. C. Rau and V. A. Parsegian, Biophys. J. 61, 260 (1992).

${ }^{3}$ A review on DNA condensation appears in: V. A. Bloomfield, Curr. Opin. Struct. Biol. 6, 334 (1996).

${ }^{4}$ E. Raspaud, M. Olvera de la Cruz, J.-L. Sikorav, and F. Livolant, Biophys. Jour. 74, 381 (1998).

${ }^{5}$ E. Raspaud, I. Chaperon, A. Leforestier, and F. Livolant, Biophys. J. 77, 1547 (1999).

${ }^{6}$ M. Drifford, J. P. Dalbiez, M. Delsanti, and L. Belloni, Ber. Bunsenges. Phys. Chem. 100, 829 (1996).

${ }^{7}$ E. Raspaud E, A. Leforestier, and F. Livolant, Biophys. J. 81, 1127 (2001).

${ }^{8}$ P. Linse and V. Lobaskin, Phys. Rev. Lett. 83, 4208 (1999).

${ }^{9}$ M. J. Stevens, Biophys. J. 80, 2075 (2001).

${ }^{10} \mathrm{~N}$. Lee N and D. Thirumalai, Macromolecules 34, 3446 (2001).

11 B. I. Shklovskii, Phys. Rev. Lett. 82, (1999) 3286.

${ }^{12}$ F. J. Solis and M. Olvera de la Cruz, J. Chem. Phys. 112, 1030 (2000).

13 T. T. Nguyen, I. Rouzina, and B. I. Shklovskii, J. Chem. Phys. 112, 2562 (2000).

${ }^{14}$ F. J. Solis and M. Olvera de la Cruz, Eur. Phys. J. E 4, 143 (2001).

15 The Bjerrum assocaition is disscused in: B. E. Conway, Ionic Interactions and Activity Behavior of Electrolyte Solutions section 7.2.3. included in B. E. Conway, J. O'M. Bockris, and E. Yeager Comprehensive Treatise of Electrochemistry; v.5. New York: Plenum Press (1983).

${ }^{16}$ A survety of the work of the Shklovskii group on charge inversion appears A.Yu. Grosberg, T.T. Nguyen, and B.I. Shklovskii, preprint cond-mat/0105140 (2001).

${ }^{17}$ M. Tanaka and A. Y. Grosberg, J. Chem. Phys. 115, 567 (2001).

18 M. Deserno and C. Holm, preprint cond-mat/0203599 (2002). 
${ }^{19}$ E. Gonzales-Tovar, M. Lozada-Cassou, and D. Henderson, J. Chem. Phys. 83, 361 (1985).

${ }^{20}$ R. Kjellander, Ber. Bunsenges, Phys. Chem. 100, 894 (1996).

21 T. T. Nguyen and B. I. Shklovskii, preprint condmat/0105078, (2001).

${ }_{22}^{2}$ R. Messina, C. Holm, and K. Kremer, Phys. Rev. Lett. 85, $872(2000)$.
${ }^{23}$ B. D. Ermi, and E. J. Amis, Macromolecules 31, 7378 (1998).

${ }^{24}$ I. Sabbagh, M. Delsanti, and P. Lisieur, Eur. Phys. J. B 12, 253 (1999).

${ }^{25}$ W. Essafi, F. Lafuma, and C.E. Williams, Eur. Phys. J. B 9, 261 (1999).

${ }^{26}$ E. Raspaud E, M. da Conceicao, and F. Livolant, Phys. Rev. Lett. 842533 (2000). 